\title{
Agreement between adolescents' and their mothers' reports of oral health- related quality of life
}

\section{Meire Coelho Ferreira(a) Daniela Goursand ${ }^{(a)}$ Cristiane Baccin Bendo(a) Maria Letícia Ramos-Jorge ${ }^{(b)}$ Isabela Almeida Pordeus(a) Saul Martins Paiva ${ }^{(a)}$}

(a) Department of Pediatric Dentistry and Orthodontics, School of Dentistry, Federal University of Minas Gerais, Belo Horizonte, MG, Brazil.

(b) Department of Health Sciences, School of Dentistry, Federal University of Jequitinhonha and Mucuri Valleys, Diamantina, MG, Brazil.
Declaration of Interests: The authors certify that they have no commercial or associative interest that represents a conflict of interest in connection with the manuscript.

Corresponding author:

Meire Coelho Ferreira

E-mail:meirecofe@ig.com.br

Received for publication on Oct 26, 2011 Accepted for publication on Jan 31, 2012

\begin{abstract}
The aim of this study was to determine the level of agreement between reports by adolescents and their mothers regarding the impact of oral health on the adolescents' quality of life. The sample consisted of 960 pairs of adolescents aged 11 to 14 years and their mothers. The Brazilian version of the Parental-Caregiver Perceptions Questionnaire (P$\mathrm{CPQ})$ and the short form of the Child Perceptions Questionnaire $\left(\mathrm{CPQ}_{11}\right.$ $\left.{ }_{14}\right)$ were administered. Agreement on the total scale and the subscales was determined through a comparison of mean values. The means of the directional and absolute differences were also determined. Agreement on each item on the group level and in the individual pairs was analyzed using the Intraclass Correlation Coefficient (ICC). The difference between the means and the directional differences were significant for the total score as well as for the functional limitation and social well-being subscales $(\mathrm{p}<0.001)$. The ICC for the total P-CPQ and $\mathrm{CPQ}_{11-14}$ scores was 0.43 . The ICC indicated weak agreement on the subscales, ranging from 0.36 to 0.40 . Agreement between reports by adolescents and their mothers regarding oral health-related quality of life (OHRQoL) was moderate, indicating that both perspectives should be considered.
\end{abstract}

Descriptors: Proxy; Mothers; Adolescent; Oral Health; Quality of Life.

\section{Introduction}

Children's perspectives on the impact of oral health on their quality of life may differ from the views of their parents or guardians. However, parents may provide complementary information because they are closely involved with their children's health, including decision making. ${ }^{1,2}$ The choice of the proxy measure should also be considered. Due to cultural and social changes, mothers currently spend less time with their children than they have in the past. However, mothers continue to be involved in caring for their children and respond reliably to questions regarding their children's health. ${ }^{3}$ As such, mothers account for the highest percentage of proxy measures employed in studies of children's health..$^{1,2,4-7}$

In a systematic review of parent-child agreement on ratings of children's oral health-related quality of life (OHRQoL), ${ }^{8}$ only three studies assessed parent-child agreement regarding children's OHRQoL. ${ }^{2,5,9}$ These studies were conducted with convenience samples in developed countries. However, there is a lack of this type of study among population-based samples. 
The aim of the present study was to determine the agreement between reports by adolescents and their mothers regarding the impact of oral conditions on adolescents' OHRQoL.

\section{Methodology}

The participants were selected from a population of 170,289 adolescents between 11 and 14 years old enrolled at 311 public and 145 private elementary schools in the city of Belo Horizonte from September 2008 to May 2009..$^{10}$ Adolescents with no systemic and/or mental developmental disorders participated in this study. The clinical dental examination was conducted during daytime hours in a private room offered by the school. The participants were clinically examined for dental caries, ${ }^{11}$ malocclu$\operatorname{sion}^{12}$ and traumatic dental injuries ${ }^{13}$ by three calibrated examiners (Kappa values for these conditions were $0.86,0.68$ and 1.00 , respectively).

The sample size was calculated to give a level of precision of $4 \%$. A $95 \%$ confidence interval (CI) and an estimated concordance of $50 \%$ were used for the calculation. Because two-step sampling was employed, a correction factor for the design effect for the cluster sampling of 1.4 was adopted to increase the accuracy. ${ }^{14}$ The minimal sample size to satisfy the parameters was estimated at 837 adolescents. The sample was increased by $20 \%$ to compensate for possible losses, for a total of 1,003 adolescents. In the first stage, public and private schools were randomly chosen in each administrative district of Belo Horizonte. In the second stage, classes were chosen at the selected schools. In the third stage, the class period (morning or afternoon) was selected, and students in each class were selected in the fourth stage.

The study received approval from the Human Research Ethics Committee of the Federal University of Minas Gerais. All participants signed terms of informed consent.

The data were collected using the Brazilian version of the Parental-Caregiver Perceptions Questionnaire (P-CPQ) and the short form of the Child Perceptions Questionnaire $\left(\mathrm{CPQ}_{11-14}\right)$, which were administered to the mothers and adolescents, respectively. ${ }^{15,16}$ These instruments include 14 items organized into four subscales:

- oral symptoms (OS) ("pain”, “mouth sores", "bad breath", "food caught in or between teeth");

- functional limitation (FL) ("taking longer than others to eat a meal", "difficulty biting or chewing food, such as fresh apple, corn on the cob or firm meat", "difficulty saying words", "difficulty eating or drinking hot or cold foods");

- emotional well-being (EWB) ("irritable or frustrated", "shy, embarrassed or ashamed", "upset") and

- social well-being (SWB) ("avoided smiling or laughing when around other adolescents", "teased or called names by other adolescents", "asked questions by other adolescents about teeth, lips, mouth or jaws”).

Each item addresses the frequency of events in the previous three months. A higher total score on the scale denotes a greater impact of oral status on the adolescent's quality of life. A 5-point scale is used, with the following options:

- "Never" = 0;

- "Once/twice" = 1;

- "Sometimes" = 2;

- "Often" = 3; and

- "Every day/almost every day" = 4..$^{2,17}$

"I don't know" responses were recorded as 0 .

Descriptive analysis was performed for the comparison of scores (paired t-test) and agreement on the group level and in the individual pairs (Intraclass Correlation Coefficient - ICC). The level of significance was set at $0.05 .^{18}$ The directional and absolute differences were calculated by subtracting the adolescent's score from the mother's score.

The ratio of the mean directional difference to the standard deviation of this difference was calculated to determine the magnitude of the differences between the reports of mothers and adolescents. For the interpretation of the magnitude of the differences, Cohen's standards were employed $0.2=$ small; $0.5=$ medium; $0.8=$ large).${ }^{19}$ The ICC was calculated for the total P-CPQ and $\mathrm{CPQ}_{11-14}$ scores, the subscale score and the score on each question to 
compare the responses given by the pairs individually. The ICC was also determined according to the independent variables. The level of agreement reflected by the ICC was categorized as follows:

- $<0.2$ (poor);

- 0.21-0.40 (fair);

- 0.41-0.60 (moderate);

- 0.61-0.80 (substantial) and

- 0.81-1.0 (excellent to perfect). ${ }^{20}$

Statistical analysis was performed using the Statistical Package for the Social Sciences (SPSS for Windows, version 15.0, SPSS Inc., Chicago, USA).

\section{Results}

Among the 1,003 questionnaires sent to the mother-adolescent pairs, 43 were excluded due to incomplete information; therefore, 960 motheradolescent pairs returned completed questionnaires $($ response rate $=95.7 \%)$. Table 1 displays the characteristics of the adolescents.

The adolescents reported experiencing a greater impact from oral health status on quality of life than was reported by their mothers. This difference was statistically significant for the total scale as well as the FL and SWB subscales ( $\mathrm{p}<0.001)$. The mean directional difference between the reports of both parties was statistically significant for the total scale as well as the FL and SWB subscales. The magnitude of these differences was small (Table 2). The absolute differences in total scale scores among the mother-adolescent pairs ranged from 0 to 35, with $76.3 \%$ achieving a score equal to or less than 10. The median score of the absolute differences was 5.0 , representing $53.1 \%$ of the scores obtained from the sample. The mean absolute differences in subscale scores ranged from 1.78 to 2.66 , with the highest score corresponding to the FL and the lowest corresponding to the SWB (Table 2). The distribution of the directional differences is displayed in Table 3. The total scale score of the P-CPQ was lower than that of the $\mathrm{CPQ}_{11-14}$ among $55.1 \%$ of the mother-adolescent pairs.

The ICC indicated weak agreement on the subscales (Table 4). Regarding gender and age, there was moderate, statistically significant agreement for the female gender and the ages of 11,12 and 13

Table 1 - Characteristics of the adolescents.

\begin{tabular}{l|c}
\hline \multicolumn{1}{c|}{ Gender } & $\mathrm{n}(\%)$ \\
\hline Male & $373(38.9)$ \\
\hline Female & $587(61.1)$ \\
\hline \multicolumn{1}{c}{ Age (years) } & $\mathrm{n}(\%)$ \\
\hline 11 & $276(28.8)$ \\
\hline 13 & $262(27.3)$ \\
\hline 14 & $220(22.9)$ \\
\hline Public & $202(21.0)$ \\
\hline Private & $\mathrm{n}(\%)$ \\
\hline
\end{tabular}

Table 2 - Mean, mean directional and absolute differences between overall and subscale P-CPQ and CPQ ${ }_{11-14} \mathrm{scores.}$

\begin{tabular}{|c|c|c|c|c|c|c|c|c|c|}
\hline & \multirow{2}{*}{$\begin{array}{l}\text { No. } \\
\text { of } \\
\text { items }\end{array}$} & \multicolumn{2}{|c|}{ Mother } & \multicolumn{2}{|c|}{ Adolescent } & \multicolumn{3}{|c|}{ Directional differences $^{a}$} & \multirow{2}{*}{$\begin{array}{c}\text { Absolute differences }^{\text {d }} \\
\text { Mean } \pm \text { SD }\end{array}$} \\
\hline & & Mean \pm SD & $\begin{array}{l}\text { Minimum- } \\
\text { maximum }\end{array}$ & Mean \pm SD & $\begin{array}{l}\text { Minimum- } \\
\text { maximum }\end{array}$ & Mean \pm SD & $p^{b}$ & $d^{c}$ & \\
\hline Total scale (0-56) & 14 & $10.16 \pm 7.86^{*}$ & $0-42$ & $11.32 \pm 7.15^{*}$ & $0-37$ & $1.16 \pm 9.02$ & $<0.001$ & 0.13 & $6.84 \pm 6.00$ \\
\hline \multicolumn{10}{|c|}{ Subscales } \\
\hline OS $(0-16)$ & 4 & $4.06 \pm 2.63$ & $0-14$ & $4.13 \pm 2.48$ & $0-14$ & $0.07 \pm 3.17$ & 0.515 & 0.02 & $2.43 \pm 2.04$ \\
\hline $\mathrm{FL}(0-16)$ & 4 & $2.88 \pm 2.98^{*}$ & $0-16$ & $3.50 \pm 2.67^{*}$ & $0-14$ & $0.61 \pm 3.51$ & $<0.001$ & 0.17 & $2.66 \pm 2.38$ \\
\hline EWB (0-12) & 3 & $1.86 \pm 2.60$ & $0-12$ & $2.00 \pm 2.25$ & $0-12$ & $0.14 \pm 3.03$ & 0.145 & 0.05 & $2.08 \pm 2.20$ \\
\hline SWB (0-12) & 3 & $1.36 \pm 2.24^{*}$ & $0-10$ & $1.69 \pm 2.11^{*}$ & $0-11$ & $0.33 \pm 2.65$ & $<0.001$ & 0.12 & $1.78 \pm 2.00$ \\
\hline
\end{tabular}

* Statistically significant differences between mothers and adolescents, $\alpha<0.05$ (paired t-test). ${ }^{\circ}$ Difference between adolescent and mother scores account for the direction of differences. ${ }^{b} \mathrm{p}$-values obtained from paired t-test. ${ }^{c}$ Standardized difference $=$ mean directional difference / standard deviation of directional differences. ${ }^{\mathrm{d}}$ Difference between adolescent and mother scores irrespective of the direction of differences. 
Table 3 - Distribution of directional differences between total and subscale P-CPQ and $C P Q_{11-14}$ scores.

\begin{tabular}{c|c|c|c|c|}
\hline \multicolumn{1}{|c|}{ Scale } & $\begin{array}{c}\text { Mother score }> \\
\text { adolescent score } \\
\mathrm{n}(\%)\end{array}$ & $\begin{array}{c}\text { Mother score }= \\
\text { adolescent score } \\
\mathrm{n}(\%)\end{array}$ & $\begin{array}{c}\text { Mother score }< \\
\text { adolescent score } \\
\mathrm{n}(\%)\end{array}$ & p-value* \\
\hline Total scale (0-56) & $365(38.0)$ & $66(6.9)$ & $529(55.1)$ & 0.01 \\
\hline \multicolumn{5}{|c|}{ Subscales } \\
\hline OS (0-16) & $403(42.0)$ & $137(14.3)$ & $420(43.7)$ & 0.01 \\
\hline FL (0-16) & $307(32.0)$ & $148(15.4)$ & $505(52.6)$ & 0.06 \\
\hline EWB (0-12) & $280(29.2)$ & $286(29.8)$ & $394(41.0)$ & 0.01 \\
\hline SWB (0-12) & $235(24.5)$ & $311(32.4)$ & $414(43.1)$ & 0.21 \\
\hline
\end{tabular}

* One-way ANOVA. years (Table 4). For all items of the scale, the ICC was statistically significant, with values in the range of 0.16 to 0.45 , but only two items reached the moderate category of strength: "Difficulty eating or drinking hot or cold foods" and "Avoided smiling or laughing when around other adolescents" (Table 4).

\section{Discussion}

Only four previous studies tested the agreement between the mother and child reports regarding the impact of oral conditions on the child's quality of life using the $\mathrm{CPQ}_{11-14}$ and P-CPQ. ${ }^{2,5,7,9}$ Mothers can provide reliable reports on the effect of oral conditions on children, or their reports can complement children's reports on the impact of oral health on quality of life. In Brazilian culture, decisions related to caring for children's health are generally the responsibility of their mothers. Although adolescents in the 11- to 14-year age group may be capable of reporting the effect of oral health on their quality of life, they do not traditionally do so. For adolescents, relationships between peers are important components of their perceptions of health and quality of life. ${ }^{21}$

There were statistically significant differences between the reports of the mothers and adolescents on the FL and SWB subscales. However, in determining the level of agreement of the pairs individually, significant moderate agreement was found for the items "difficulty eating or drinking hot or cold foods" (FL subscale) and "avoided smiling or laughing when around other adolescents" (SWB). It should be stressed that these items seem to be the most easily observed aspects among the items on the FL and SWB subscales. The sensation of heat or cold is readily externalized, and the way an individual behaves in socially favorable and unfavorable settings is readily detectable. Parents are more capable of judging problems that are externalized by the child and are less accurate at judging internalized problems. ${ }^{22}$

It is reasonable to expect parents' knowledge of their children to be limited, especially with respect to activities and relationships outside the family setting and internal feelings. ${ }^{2}$ A systematic review of the relationship between a parent's proxy measure and children's reports on quality of life shows that the level of agreement appears to be dependent on the domain investigated, with adequate agreement on domains that reflect physical activity, function and symptoms and poor agreement on domains that reflect social and emotional issues. ${ }^{23}$ In the present study, the EWB was the subscale with the weakest agreement when considering the pairs individually.

Although the mean differences between the reports of mothers and adolescents were statistically significant for the total score as well as the FL and SWB subscales, the magnitude of these differences was small. The distribution of directional differences suggests that mothers tend to underreport the impact of oral conditions on their children's quality of life.

The moderate level of agreement between the mothers and adolescents for the total score contrasts with the substantial agreement reported by other studies. ${ }^{2,5}$ One study found moderate agreement on subjective subscales and substantial agreement on objective subscales, ${ }^{5}$ whereas in the present study, 
Table 4 - Agreement between mothers and adolescents considering overall and subscale scores, overall score according to age and gender of adolescent and each item of the P-CPQ and $C P Q_{11-14}$ scales.

\begin{tabular}{|c|c|c|}
\hline Scale/subscales & ICC & $(95 \% \mathrm{Cl})$ \\
\hline Total scale & 0.43 & $(0.35-0.50)$ \\
\hline \multicolumn{3}{|l|}{ Subscales } \\
\hline - OS & 0.37 & $(0.29-0.45)$ \\
\hline - $\mathrm{FL}$ & 0.36 & $(0.27-0.43)$ \\
\hline - EWB & 0.37 & $(0.28-0.44)$ \\
\hline \multirow[t]{2}{*}{ - SWB } & 0.40 & $(0.32-0.47)$ \\
\hline & \multicolumn{2}{|c|}{$p<0.001$} \\
\hline Gender & ICC & p-value \\
\hline Male & 0.20 & 0.015 \\
\hline Female & 0.53 & $<0.001$ \\
\hline Age (years) & ICC & p-value \\
\hline 11 & 0.46 & $<0.001$ \\
\hline 12 & 0.57 & $<0.001$ \\
\hline 13 & 0.42 & $<0.001$ \\
\hline 14 & 0.11 & 0.199 \\
\hline Scale Item & ICC & $p$-value \\
\hline \multicolumn{3}{|l|}{ OS } \\
\hline - Pain & 0.35 & $<0.001$ \\
\hline - Mouth sores & 0.32 & $<0.001$ \\
\hline - Bad breath & 0.34 & $<0.001$ \\
\hline - Food caught in or between teeth & 0.16 & $=0.004$ \\
\hline \multicolumn{3}{|l|}{$\mathrm{FL}$} \\
\hline - Taking longer than others to eat a meal & 0.31 & $<0.001$ \\
\hline $\begin{array}{l}\text { - Difficulty biting or chewing food, such as fresh apple, corn on the } \\
\text { cob or firm meat }\end{array}$ & 0.28 & $<0.001$ \\
\hline - Difficulty saying words & 0.35 & $<0.001$ \\
\hline - Difficulty eating or drinking hot or cold foods & 0.45 & $<0.001$ \\
\hline \multicolumn{3}{|l|}{ EWB } \\
\hline - Irritable or frustrated & 0.28 & $<0.001$ \\
\hline - Shy, embarrassed or ashamed & 0.29 & $<0.001$ \\
\hline - Upset & 0.27 & $<0.001$ \\
\hline \multicolumn{3}{|l|}{ SWB } \\
\hline - Avoided smiling or laughing when around other adolescents & 0.43 & $<0.001$ \\
\hline - Teased or called names by other adolescents & 0.37 & $<0.001$ \\
\hline - Asked questions by other adolescents about teeth, lips, mouth or jaws & 0.22 & $<0.001$ \\
\hline
\end{tabular}

agreement was only fair on all subscales. Brazil is a developing country, and a substantial portion of the Brazilian population is economically underprivileged, which has significant consequences on health and education and may affect proxy measures. In the present study, the majority of the sample was en- rolled in public school, a variable that may be used as an alternative indicator of low socioeconomic status. $^{24}$ The adolescents reported a greater impact of oral health on their quality of life than their mothers did, as described in studies involving Canadian children. ${ }^{2,5}$ A study conducted by Benson et al. ${ }^{7}$ with 
English children found that mothers over-reported the impact of malocclusion on their children's quality of life with regard to the emotional well-being subscale. The same behavior was observed in a study assessing oral health-related quality of life among American adolescents with ectodermal dysplasia, but differences in the total score and subscale scores were not statistically significant, which may have been due to the small sample size. ${ }^{25}$

One may expect greater agreement between parents and children with age because greater verbal skills may improve a child's ability to describe his/her experiences and emotions. ${ }^{23}$ However, this phenomenon was not found in the study. There was poor agreement between mothers and 14-year-olds, suggesting that physical and emotional changes in the course of adolescence are laden with satisfactions and heartaches, making young individuals more introspective. These developments may have repercussions on adolescents' family relationships. Moreover, older adolescents spend more time away from their parents' supervision and therefore share their experiences less frequently. ${ }^{26}$ The findings of the present study corroborate those in a Canadian study, which found lower levels of agreement among older adolescents. With regard to gender, mother-adolescent agreement was moderate among female adolescents and poor among male adolescents. However, the Canadian study reported a lower agreement between mothers and female adolescents. ${ }^{5}$ In the present study, a likely explanation for this result is that the sample consisted of more females than males. According to the census data

\section{References}

1. Jokovic A, Locker D, Stephens M, Kenny D, Tompson B, Guyatt G. Measuring parental perceptions of child oral health-related quality of life. J Public Health Dent. 2003 Spring;63(2):67-72.

2. Jokovic A, Locker D, Guyatt G. How well do parents know their children? Implications for proxy reporting of child healthrelated quality of life. Qual Life Res. 2004 Sep;13(7):1297307.

3. Landgraf JM, Abetz LN. Functional status and well-being of children representing three cultural groups: initial self-reports using the CHQ-CF87. Psychol Health. 1997 Sep;12(6):839-54.
(2008/2009), girls account for $50.2 \%$ and boys account for $49.8 \%$ of the children regularly enrolled in the schools in Belo Horizonte. ${ }^{10}$ In this study, however, only those students in class on the day of the data collection were selected, which constitutes a limitation of the study. Thus, it was not possible to maintain the proportion of boys to girls.

Additional reports by parents are important for obtaining a broad perspective, and these reports contribute toward decision making with regard to children's health. Valuable information may be lost if one report is prioritized over the other. ${ }^{5}$ Parents' perceptions regarding their children's oral health facilitate children's access to dental services because parents may perceive treatment needs in their children before any form of care is considered. Overall, the mothers and adolescents had moderate agreement on OHRQoL.

\section{Conclusion}

The views of both parties should be considered to obtain a comprehensive view of the impact of oral health on adolescents' quality of life. These views may offer a broader basis for clinical decisions and for guiding oral health policies around the world, including in Brazil.

\section{Acknowledgments}

This study was supported by the National Council for Scientific and Technological Development (CNPq), the Ministry of Science and Technology and the State of Minas Gerais Research Foundation (FAPEMIG), Brazil.
4. Loonen HJ, Derkx BHF, Koopman HM, Heymans HSA. Are parents able to rate the symptoms and quality of life of their offspring with IBD? Inflamm Bowel Dis. 2002 Jul;8(4):270-6.

5. Jokovic A, Locker D, Stephens M, Guyatt G. Agreement between mothers and children aged 11-14 years in rating child oral health-related quality of life. Community Dent Oral Epidemiol. 2003 Oct;31(5):335-43.

6. Wilson-Genderson M, Broder HL, Phillips C. Concordance between caregiver and child reports of children's oral healthrelated quality of life. Community Dent Oral Epidemiol. 2007 Aug;35(Suppl 1):32-40. 
7. Benson P, O’Brien C, Marshman Z. Agreement between mothers and children with malocclusion in rating children's oral health-related quality of life. Am J Orthod Dentofacial Orthop. 2010 May;137(5):631-8.

8. Barbosa TS, Gavião MBD. Oral health-related quality of life in children: part III. Is there agreement between parents in rating their children's oral health-related quality of life? A systematic review. Int J Dent Hyg. 2008 May;6(2):108-13.

9. Johal A, Cheung MYH, Marcenes W. The impact of two different malocclusion traits on quality of life. Br Dent J. 2007 Jan;202(2):E2.

10. Prefeitura de Belo Horizonte - $\mathrm{PBH}$ [home page]. Belo Horizonte: Census data of school; 2007 [cited 2009 May 27]. Available from: http:// www.pbh.gov.br.

11. World Health Organization. Oral Health Surveys. Basic Methods. 4th ed. Geneva: World Health Organization; 1997. 66 p.

12. Cons NC, Jenny J, Kohout FJ. DAI: the dental aesthetic index. Iowa City: Iowa College of Dentistry, University of Iowa; 1986. $17 \mathrm{p}$.

13. Andreasen JO, Andreasen FM, Andersson L. Textbook and color atlas of traumatic injuries to the teeth. $4^{\text {th }}$ ed. Copenhagen: Munskgaard; 2007.897 p.

14. Kirkwood BR, Stern J. Essentials of Medical Statistics. $2^{\text {nd }}$ ed. London: Wiley-Blackwell; 2003. 512 p.

15. Goursand D, Paiva SM, Zarzar PM, Pordeus IA, Grochowski $\mathrm{R}$, Allison PJ. Measuring parental-caregiver perceptions of child oral health-related quality of life: psychometric properties of the Brazilian version of the P-CPQ. Braz Dent J. 2009;20(2):169-74.

16. Torres CS, Paiva SM, Vale MP, Pordeus IA, Ramos-Jorge ML, Oliveira AC, et al. Psychometric properties of the Brazilian version of the Child Perceptions Questionnaire $\left(\mathrm{CPQ}_{11-14}\right)$ short forms. Health Qual Life Outcomes. 2009 May;7:43.
17. Jokovic A, Locker D, Stephens M, Kenny D, Tompson B, Guyatt G. Validity and reliability of a questionnaire for measuring child oral-health-related quality of life. J Dent Res. 2002 Jul;81(7):459-63.

18. Streiner DL, Norman GR. Health measurement scale: a practical guide to their development and use. 3rd ed. New York: Oxford; 2003. 432 p.

19. Cohen J. A power primer. Psychol Bull. 1992 Jul;112(1):155-9.

20. Landis JR, Koch GG. The measurement of observer agreement for categorical data. Biometrics. 1977 Mar;33(1):159-74.

21. Biazevic MGH, Rissotto RR, Michel-Crosato E, Mendes LA, Mendes MOA. Relationship between oral health and its impact on quality of life among adolescents. Braz Oral Res 2008 Jan-Mar;22(1):36-42.

22. Achenbach TM, McConaughy SH, Howell CT. Child/adolescent behavioural and emotional problems: implications of cross-informant correlations for situational specificity. Psychol Bull. 1987 Mar;101(2): 213-32.

23. Eiser C, Morse R. Can parents rate their child's health-related quality of life? Results of a systematic review. Qual Life Res. 2001;10(4):347-57.

24. Piovesan C, Pádua MC, Ardenghi TM, Mendes FM, Bonini GC. Can type of school be used as an alternative indicator of socioeconomic status in dental caries studies? A crosssectional study. BMC Med Res Methodol. 2011 Apr;11:37. doi:10.1186/1471-2288-11-37.

25. Kohli R, Levy S, Kummet CM, Dawson DV, Stanford CM. Comparison of perceptions of oral health-related quality of life in adolescents affected with ectodermal dysplasias relative to caregivers. Spec Care Dentist. 2011 May-Jun;31(3): 88-94.

26. Wallander JL, Schmitt M, Koot HM. Quality of life measurement in children and adolescents: issues, instruments, and applications. J Clin Psychol. 2001 Apr;57(4):571-85. 